\title{
Resilience Throughout Life: Investigating Processes of Coping with Many Adversities
}

\author{
Juliana Mendanha Brandão*1 and Elizabeth do Nascimento ${ }^{2}$ \\ ${ }^{1}$ Psicologia, Pontifical Catholic University of Minas Gerais, Brazil \\ ${ }^{2}$ Psicologia, Federal University of Minas Gerais, Brazil
}

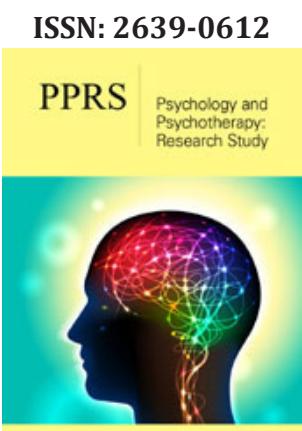

*Corresponding author: Juliana $M$ Brandão, Department of Psicologia, PUCMinas, Brazil

Submission: 㘹 February 15, 2021

Published: 侮 February 22, 2021

Volume 4 - Issue 4

How to cite this article: Juliana Mendanha Brandão, Elizabeth do Nascimento. Resilience Throughout Life: Investigating Processes of Coping with Many Adversities. Psychol Psychother Res Stud. 4(4). PPRS. 0005942021. DOI: 10.31031/PPRS.2021.04.000594

Copyright@ Juliana M Brandão, This article is distributed under the terms of the Creative Commons Attribution 4.0 International License, which permits unrestricted use and redistribution provided that the original author and source are credited.

\begin{abstract}
This article presents a case study that contributes to the field of research on resilience. It has the trajectory-based approaches as its starting point, aiming to obtain the description and the understanding of the processes of coping with adversities throughout life, identifying different coping trajectories, differentiating them according to the outcome of each one, and revealing resilience factors. Through phenomenological analysis, it was possible to raise nine adverse events faced by a person and six types of coping trajectories: resistance; minimal-impact resilience; emergent resilience; gradual recovery with turning point, with residual pain; mild-to-moderate persistent distress and delayed dysfunction with gradual improvement and turning point, with residual pain. The proposed design proved to be promising for understanding individual resilience as a complex, dynamic, and contextual process.
\end{abstract}

Keywords: Resilience; Coping; Adaptability; Psychological stress

\section{Introduction}

Throughout life, people experience stressful or traumatic situations, which require coping efforts. Studying resilience implies trying to understand how successful processes of coping with these situations occur, as well as what factors contribute to their results [1]. As of the 2010s, the field of research on psychological resilience was enriched by studies of trajectory-based approaches that investigated the clinical consequences of stress and potentially traumatic events [2]. In these approaches, longitudinal designs and statistical models of data reading are used to identify distinct groups of subjects according to their trajectories of coping with adversities. Different types of trajectories are identified graphically from the statistical analysis of data related, for example, to physical and mental health, wellbeing, psychopathological symptoms, life satisfaction, and positive and negative affects. These data are measured some time before the subject faces the stressful event, during the coping period, and after the end of the adversity, whether it is acute or chronic $[3,4]$. These studies have identified that people subject to adversities may have minimal-impact resilience (which describes a relatively stable, healthy adjustment reaction after the acute stressful event); emergent resilience (when, after the end of chronic adversities, the positive outcome emerges); stress resistance(when the person remains healthy in the face of the adversity); chronic dysfunction (when the person does not recover after the end of adversity); delayed dysfunction (when the dysfunctions emerge a while after the end of the stressful event); post-event growth (when the person becomes healthier than before after facing his or her adversity); in addition to other trajectories. The studies of these approaches represent an advance in the field of research on resilience because they show that people with similar outcomes after facing a certain adversity may have done it in different ways. This results in overcoming the binary view of resilience in which a person is classified only as resilient or non-resilient, depending only on the assessment performed after facing an adversity. This assessment by results ends up giving resilience a trait conception and disregarding the process the person experienced.

When studying trajectory-based approaches, researchers usually seek to identify how different people face the same adversities. Seeking to advance in the understanding of the phenomenon of resilience and the processes of coping with adversities, we investigated how the same person faces different adversities throughout life. The focus shifted from interpersonal to intrapersonal. We defined resilience as a successful process of coping with adversities that 
can be achieved through several possible trajectories. We chose to assess the result using the "positive mental health" construct that goes beyond the absence of mental disorders and includes a state of well-being [5]. A woman who had faced several adversities in her life was chosen for this study and a qualitative research about her life story was carried out through the methodology of the case study.

\section{Case Presentation}

The woman investigated was 45 years old at the time of the research and had faced the following identified adversities: bullying; appendicitis; brother's anorexia; lymphoma; a treatment to get pregnant which resulted in three miscarriages; conflicts at work; and her father's cancer and subsequent death. In-depth interviews were conducted, and the data of the interviews were analyzed according to the phenomenological method. In this analysis, six types of coping trajectories were identified: resistance to stress; minimal-impact resilience; emergent resilience; gradual recovery with turning point, with residual pain; mild persistent distress; and delayed dysfunction with gradual improvement and turning point, with residual pain. The positive factors, associated with the positive outcomes of the coping processes, were recurrently recognized: the need for emotional balance; the need to be supported by the family; family support; hardiness; direct coping; and her expectations. The first two were triggers of emotional regulation. Expectations had both positive and negative effects. When this woman had an expectation that certain adversity was normal or that her suffering was expected, she had a good result in relation to that adversity. When she built highly positive expectations about something and it did not go as expected, her suffering was great, and the result of the coping process was not positive. Negative factors, associated with negative outcomes of the coping process, were also identified: fear and feeling of being harmed. This last factor had already been identified by coping theorists and is related to less efficient coping strategies.

The way the respondent faced each adversity was analyzed separately, as well as her current situation after all her coping processes. In general, when assessing positive mental health, it was considered that the woman who was studied was resilient. However, we realized that two adversities that she faced (the loss of her father and her last miscarriage) still generated suffering, although it did not cause any dysfunctions. This made us assess the outcomes of her coping considering her life story in general and facing each adversity separately. It was identified that, at some points in her life, she had conflicting feelings in relation to different sectors of life. During the period when she had conflicts at work, for example, she was suffering a lot and with depressive symptoms caused by this event, while she was incredibly happy because she had just gotten married.

\section{Discussion}

This study design allowed us to verify that the same person faces adversity throughout life in different ways, achieving different results in relation to each coping process. There is no resilience as a personality trait, producing consistent results, being favorable to any coping processes. There are several factors that affect the results: factors related to the adversity itself and factors related to the person facing the stressful event. And the way of perceiving adversities also affects how they will be faced. It is possible for a person to perform well in relation to some stressful events, but not to all of them. It is possible that one may have a positive mental health, but that same person may have some suffering in relation to a certain event. The way in which a person is at a certain point in his or her life is the result of several coping processes in which different adversities are faced, sometimes at the same time. For this reason, it is possible that studies that investigate resilience in relation to only one specific adverse event may have distorted results. A person can be categorized as resilient or dysfunctional in relation to an adversity when, in fact, other events may be related to that outcome. Qualitative studies make it possible to grasp the factors or conditions that contribute to the outcomes and help to consolidate the concept of resilience as a complex, dynamic, and contextual process.

\section{Acknowledgement}

Prof Juliana M Brandão conducted this study while taking her doctorate and Prof. Elizabeth Nascimento was her advisor.

\section{References}

1. Brandão JM and Nascimento E (2019) Psychological resilience: From the first phase to trajectory-based approaches. Memorandum 36: 1-31.

2. Levy GIR, Huang SA, Bonanno GA (2018) Trajectories of resilience and dysfunction following potential trauma: A review and statistical evaluation. Clin Psychol Rev 63: 41-55.

3. Bonanno GA, Diminich ED (2013) Annual research review: Positive adjustment to adversity- trajectories of minimal-impact resilience and emergent resilience. J Child Psychol Psychiatry 54(4): 378-401.

4. Infurna FJ, Luthar SS (2017) Parent's adjustment following the death of their child: Resilience is multidimensional and differs across outcomes examined. J Res Pers 68: 38-53.

5. Machado WL, Bandeira DR (2015) Positive mental health scale: Validation of the mental health continuum-Short form. Psico-USF 20(2): 259-274.

For possible submissions Click below: 